\title{
Ultrasound Transmission System for TDOA Localization with Unsynchronized Beacons
}

\author{
Alexander Ens ${ }^{1}$, Joan Bordoy ${ }^{2}$, Johannes Wendeberg ${ }^{2}$, Christian Schindelhauer ${ }^{2}$, Leonhard M. Reindl ${ }^{1}$ \\ ${ }^{1}$ Department of Microsystems Engineering, University of Freiburg, Georges-Köhler-Allee 106, 79110 \\ Freiburg, Germany, \{alexander.ens, reindl\}@imtek.uni-freiburg.de \\ 2 Department of Computer Science, University of Freiburg, Georges-Köhler-Allee 51, 79110 Freiburg, \\ \{bordoy, wendeber, schindel\}@informatik.uni-freiburg.de
}

\section{Summary}

Indoor localization based on time difference of arrival (TDOA) has been recently a promising field of study. We consider the previously unsolved problem of locating a moving target receiver by using unsynchronized stationary beacons without requirement of manual calibration. Thus, the received signals and their time of arrival (TOA) have to be assigned to a beacon.

We present an ultrasound transmission system approach which is robust against multipath and estimating the reception time with high accuracy. In order to avoid interference by echoes the packet size is reduced by using two frequencies in Orthogonal Frequency Division Multiplex (OFDM). Further, the transmission system enables distinction of the beacons, as the ultrasound signals are used both for localization and for information transmission.

\section{Introduction}

In our everyday life it is important to know the actual position of things. The interests in localization services are growing and there are many possible applications (e.g. as navigation of shopping carts in super markets). Localization systems based on ultrasound are very cost effective, have a low complexity and simple hardware compared to radio frequency systems [1].

The disadvantage of ultrasound is the absorption and therefore the attenuation of the transmitted signal by the air. Further the attenuation increases quadratic with the frequency $[2,3]$. Hence, we use low frequency for the transmission within $40 \mathrm{kHz}$.

In this approach, multiple unsynchronized beacons are used to track the position of a moving receiver without requirement of manual calibration. Local optimization algorithms and statistical approaches are used to estimate the initial parameters of the scenario (sender positions, intervals...), which are afterwards used in recursive state estimation (unscented Kalman filter and particle filter). In order to estimate the initial values the receiver is required to stop in certain positions receiving at least one signal burst from every sender. Once the initial values are estimated the receiver can move continuously.

Beacons can be distinguished by giving each transmitter a different frequency band for the data communication. However, broadband receivers are very expensive and the frequency bands are limited. Another robust modulation scheme is the phase shift keying (PSK) [4], which includes the information in the phase. Moreover, the frequency shift keying (FSK) can also be used for data modulation, though the bit error rate (BER) is higher than for PSK or m/4-QPSK [5].

To increase the data rate, modern transmission systems use orthogonal frequency division multiplex (OFDM) to spread the data stream onto multiple carrier [6]. The carriers are orthogonal and each can be modulated separately. We use OFDM with two carrier frequencies to achieve short pulse length.

TDOA has been often used to track the position of a moving sender using stationary receivers. The algorithms used are squared or maximum likelihood estimators [7], particle filters [8, 9] or Kalman filters [10, 11]. We consider the inverted scenario where a moving receiver is located. Wendeberg et al. [12] and Bordoy et al. [13] show the feasibility of reference and calibration free localization systems with TDOA. As a result, the mobile receivers have no information about the positions of the beacons and themself. Further, the 
beacons are unsynchronized and send in constant interval a pulse. Hence, the receiver has to estimate the positions of the beacons and their own position.

\section{System Description}

The localization system consists of installed unsynchronized ultrasound senders on the ceiling and mobile devices with ultrasound receivers. The senders have only a simplex ultrasound communication and transmit in constant intervals short packages. Further, the packages include the identification number (ID) of the sender and the temperature as data. Fig. 1 shows the principle environment of the localization system. The decentralized setup of our localization system works without a central control unit and is easy to install. The parameters of the system are estimated online in the application.
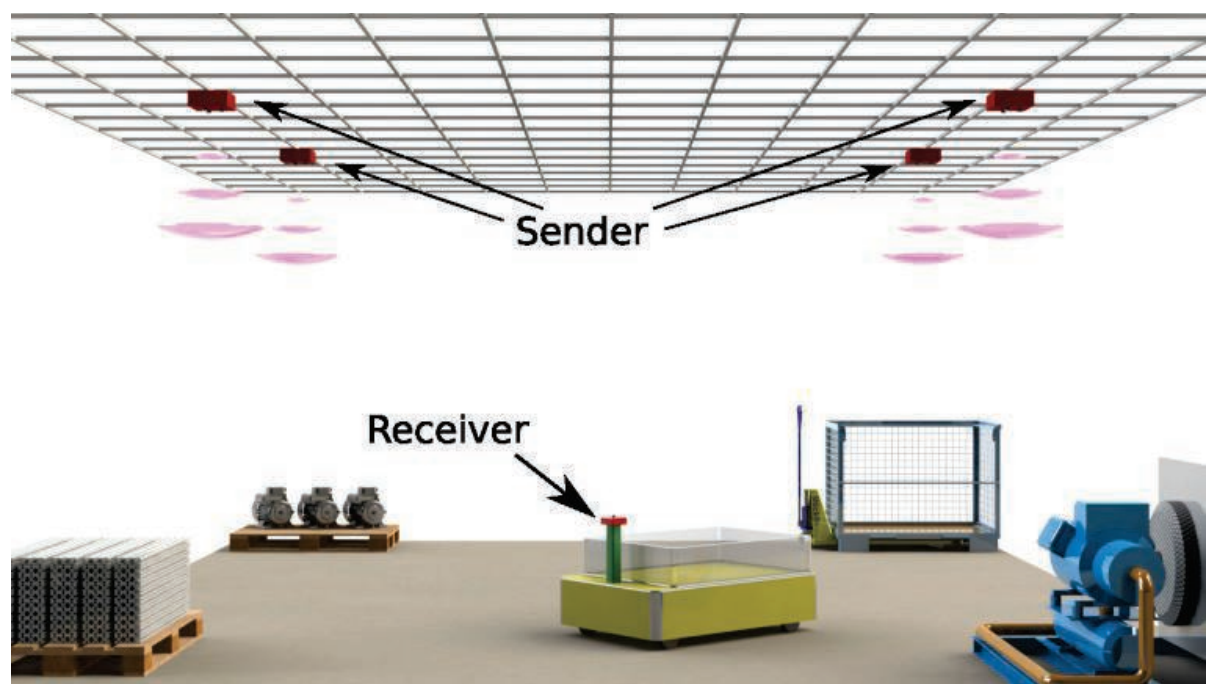

Fig. 1: Visualization of the localization environment. The senders are mounted on the ceiling and transmit the identification in constant intervals. The receiver is mobile and the position is estimated with the measured TDOA values.

The sender is designed to be powered by photovoltaic. Therefore, the signal is generated by a low power microcontroller. The power consumption is about $10 \mathrm{~mW}$ for a transmission of 1 packet per second. Hence, the senders can be powered by a indoor photovoltaic cell with $98 \mathrm{~cm}^{2}$ (about $10 \times 10 \mathrm{~cm}^{2}$ ) [14].

The data is coded and divided into two data streams. Further, the data is mapped by $\pi / 4-$ DQPSK on the both carrier frequencies $\left(f_{r 0}=38.8 \mathrm{kHz}\right.$ and $\left.\mathrm{f}_{\mathrm{r} 1}=40.8 \mathrm{kHz}\right)$ and the digital-toanalog-converter (DAC) generates the analog signal for the piezoelectric transducers. Fig. 2 shows the schematic function and Fig. 3 shows a photo of the sender. The transfer function in Fig. 2 demonstrates the possible transmission bandwidth of the piezoelectric transducers.

The receiver includes the analog signal condition and the signal processing with a microcontroller. Therefore, the piezo-electric transducer receives the transmitted ultrasound signal on both carrier frequencies. Further, the power meters triggers the microcontroller to digitize the received signal by an analog-to-digital converter (ADC). Moreover, the signal processing includes the separation of the two data carrier frequencies, the estimation of the synchronization (the time of arrival) and the estimation of the data. Fig. 2 shows the schematic function and Fig. 3 shows a photo of the receiver. The receiver is matched to receive both carrier frequencies with same amplitude to ensure equal dynamic range of the ADC for both carrier frequencies [15].

The synchronization point is the center of the first frame. Therefore, the variance of the estimated phase difference $\varphi_{D}$ is calculated by

$$
n_{\text {snyc }}=\arg \max _{n} \sum_{x=1}^{M}\left(\varphi_{D}(x+n)-\bar{\varphi}_{D}(n)\right) \varphi_{D r}(x)
$$

As a result, a theoretical synchronization accuracy of $1.5 \cdot 10^{-7} \mathrm{~s}$ is possible with an unbiased maximum likelihood estimator [16]. 

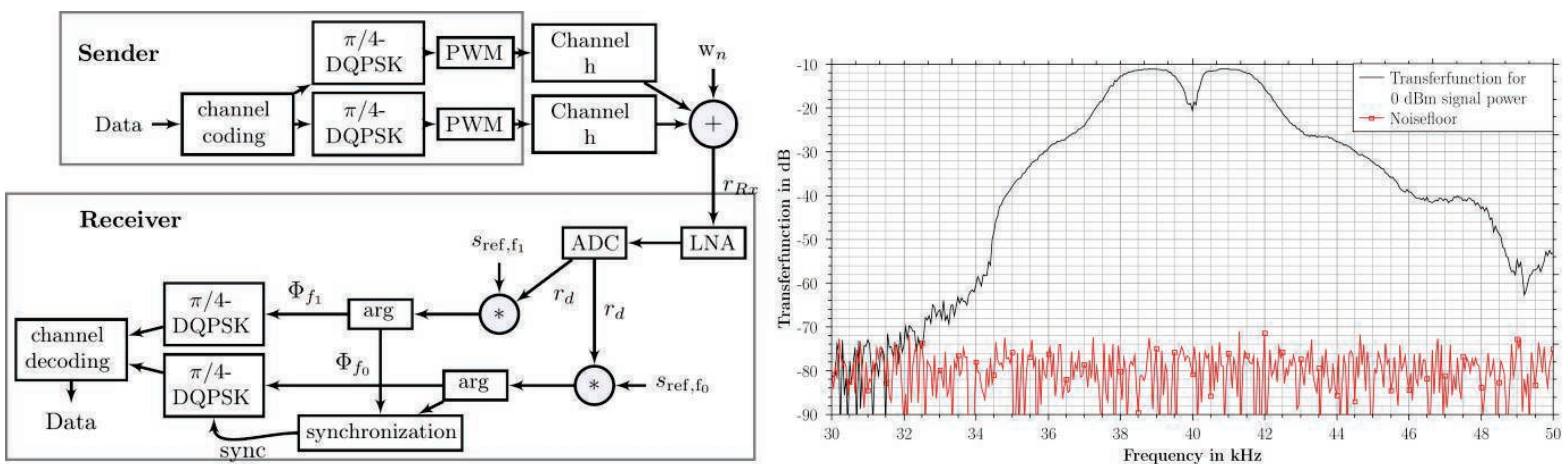

Fig. 2: Left: Schematic of the ultrasound transmission system. The micro-controller generates the modulated signal and drives the piezoelectric transducers with the PWM. Right: Plot of the measured transfer function from the sender to the receiver.
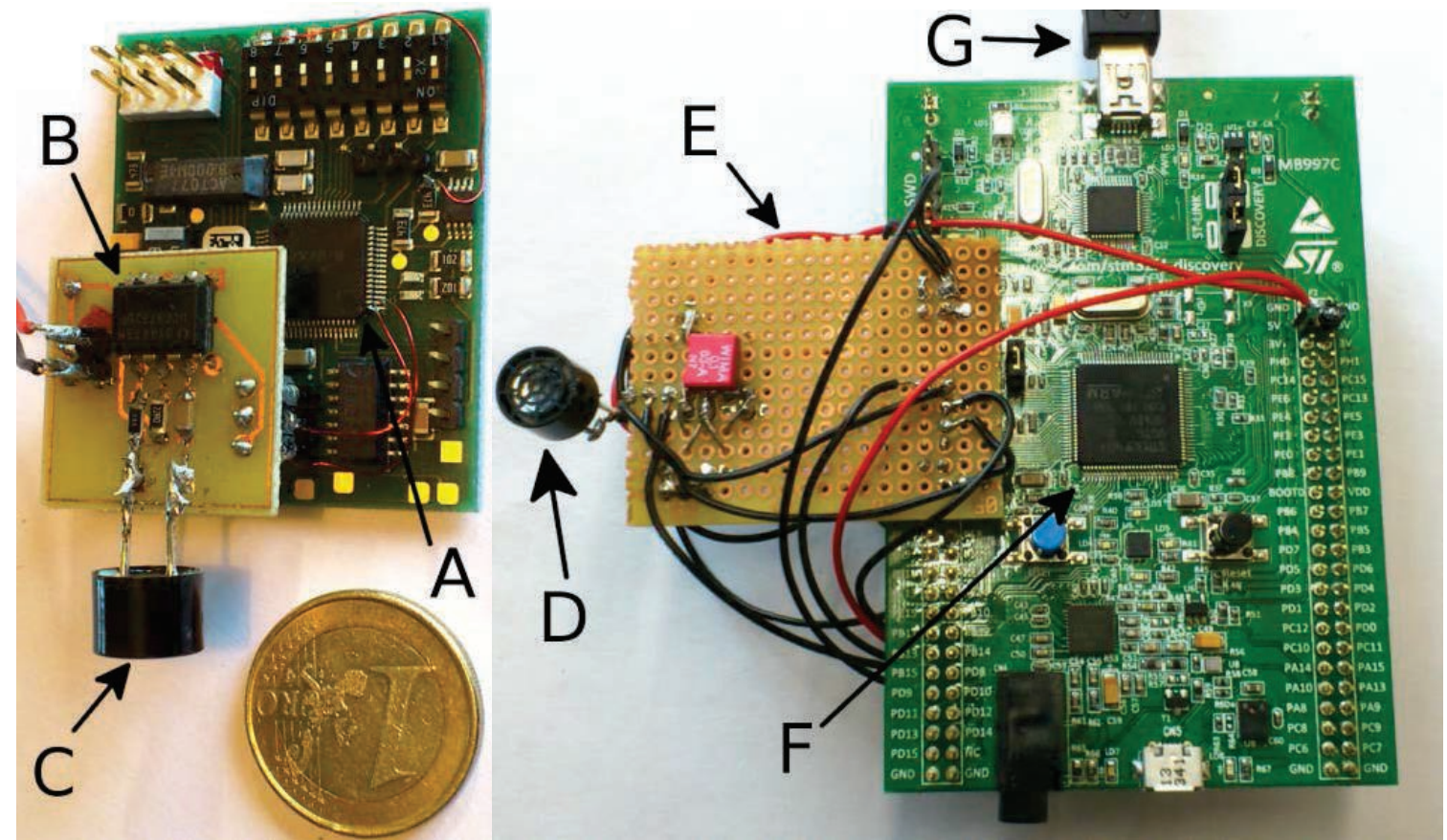

Fig. 3: Left: Photo of the sender with the microcontroller $(A)$, the power amplifier $(B)$ and the piezo-electric transducer. Right: Photo of the receiver with the piezo-electric transducer (D), the analog board (E), the ARM microcontroller STM32F407 (F) and the programming interface.

Once the reception times are precisely estimated by the system described above, the pose of a moving receiver can be tracked using recursive state estimation algorithms, as proved in [13]. However, their performance depends highly upon the knowledge of the initial values of the state. Consequently, it is mandatory to do the localization in two phases: the calibration phase, which estimates all the variables of the scenario and the tracking phase, which tracks the position of a continuously moving receiver.

The localization scenario consists of B stationary senders which are placed randomly at unknown positions $\mathrm{S}_{\mathrm{j}}(1 \leq \mathrm{j} \leq \mathrm{B})$ in a two-dimensional Euclidean space. Every sender emits discrete signals at regular points in time at a fixed interval $l_{j}$. The interval may differ from sender to sender. The sending time of the $k_{j}$-th signal at sender position $S_{j}$ is then described by

$$
t_{k_{j} j}=t_{0 j}+k_{j} I_{j} \quad\left(k_{j}>0\right) .
$$

The receiver $\mathbf{M}$ moves with a random trajectory in the two-dimensional Euclidean space. Furthermore, we assume that a $k_{j}$-th signal of sender $S_{j}$ propagates in a straight line from the sender to the receiver and is received at time point 


$$
T_{k_{j j} j}=\frac{1}{c}\left\|M-S_{j}\right\|+t_{k_{j j} j},
$$

where $c$ is the signal velocity and $\|\cdot\|$ denotes the Euclidean norm. The senders are assumed to be unsynchronized, i.e the intervals $I_{j}$ and the initial send time $t_{0 j}$ varies from sender to sender. Consequently, there is an unknown time offset which relates to the send time between the senders $y$ and $j$

$$
\delta_{y j}=t_{0, y}-t_{0, j}=\left(t_{k_{y}, y}-k_{y} I_{y}\right)-\left(t_{k_{j}, j}-k_{j} I_{j}\right) .
$$

Since the offsets are transitive, only $B-1$ offsets need to be estimated relative to one sender.

Considering the case where the receiver is continuously moving, signals are received at different positions. This results in the following hyperbolic equation in which two signals, originating from two different senders $\boldsymbol{S}_{y}$ and $\boldsymbol{S}_{j}$, are received at the positions $\boldsymbol{M}_{k_{y} y}$ and $\boldsymbol{M}_{\boldsymbol{k}_{j} j}$

$$
\frac{1}{c}\left(\left\|M_{k_{y} y}-S_{y}\right\|-\left\|M_{k_{j} j}-S_{j}\right\|\right)=\Delta t_{y j}+\delta_{y j},
$$

where $\Delta t_{y j}$ represents the unsynchronized time difference of arrival of the two signals originated by $S_{y}$ and $S_{j}$, which may be calculated based on the reception times and the intervals as

$$
\Delta t_{i j}=\left(T_{k_{y}, y}-T_{k_{j}, j}\right)-\left(T_{y} \boldsymbol{I}_{y}-k_{j} \boldsymbol{I}_{j}\right) .
$$

\section{Experimental Results}

We measured the accuracy of the distance measurement between the receiver and one sender to determine the performance of the ultrasound system. Therefore, we put the sender and receiver pair in a straight line and send in a constant interval the same package. The receiver demodulates the data and measure the TDOA between the packages. Fig. 4 left shows the TDOA measurement error for a SNR of $10 \mathrm{~dB}$. Although, the measurement error depends on the synchronization of the frame. Hence, for stationary measurements without moving, the TDOA errors are the synchronization errors. Fig 8 right shows the histogram of the synchronization errors. As a result, the variance of the synchronization error is about $1.43 \cdot 10^{-12} \mathrm{~s}$ and the standard deviation $1.19 \mu \mathrm{s}$. Which results in a distance error of $0.4 \mathrm{~mm}$ for a signal velocity of $340 \mathrm{~m} / \mathrm{s}$. Though, the standard deviation of a $500 \mathrm{kHz}$ sampled signal is about $5.8 \cdot 10^{-7} \mathrm{~s}$ respectively $0.2 \mathrm{~mm}$ [16]. Thus, the measurement system limits the precision to $0.2 \mathrm{~mm}$.
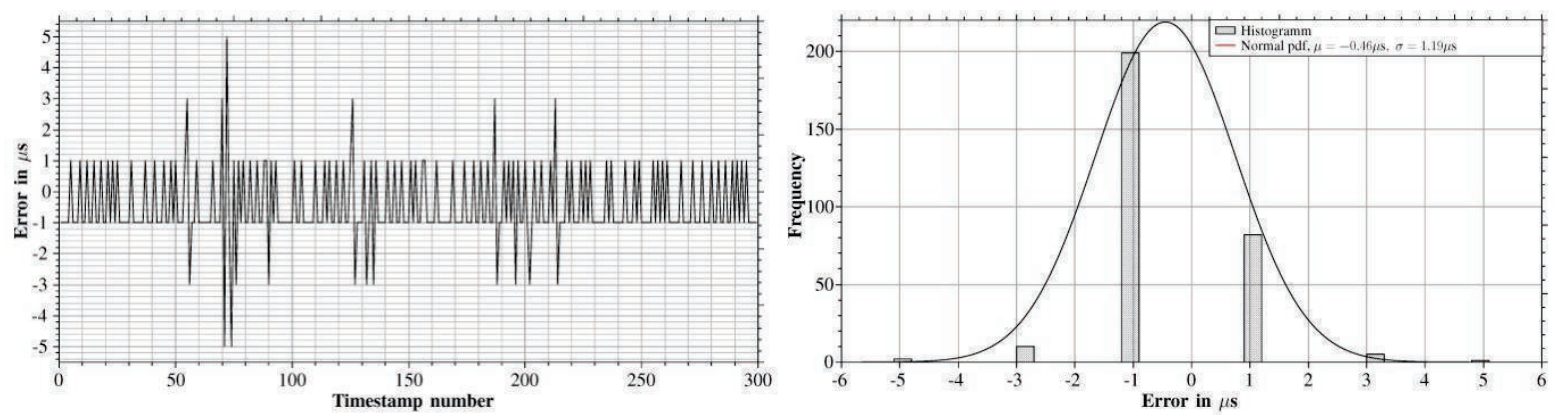

Fig. 4: Left: Error over the received timestamp number. Right: Histogram of the synchronization errors. A normal distribution with $\sigma=1.19 \mu \mathrm{s}$ and a mean of $\mu=0.46 \mu \mathrm{s}$ is fitted to the error histogram.

The second experiment is a simulation with measured parameters, where the sender positions are assumed to be known with a mean error of $0.60 \mathrm{~m}$. First, assuming these positions are correct, the receiver positions and the offsets are estimated. Afterwards, the estimated receiver positions and offsets and the known sender positions are used to initialize 
the algorithm, which corrects them. The result of this correction is shown in Fig. 5. In this experiment the receiver stops 6 times in order to increase the number of constrains and have a uniquely determined system of equations. The red points indicate the estimated positions of the senders and the lines to the points are the improvements of the position accuracy after each iteration.

The mean error of the estimated receiver positions after the last iteration is $5.3 \mathrm{~cm}$ and the mean error of the estimated sender positions is $5.6 \mathrm{~cm}$. The offsets mean error is $0.11 \mathrm{~ms}$.

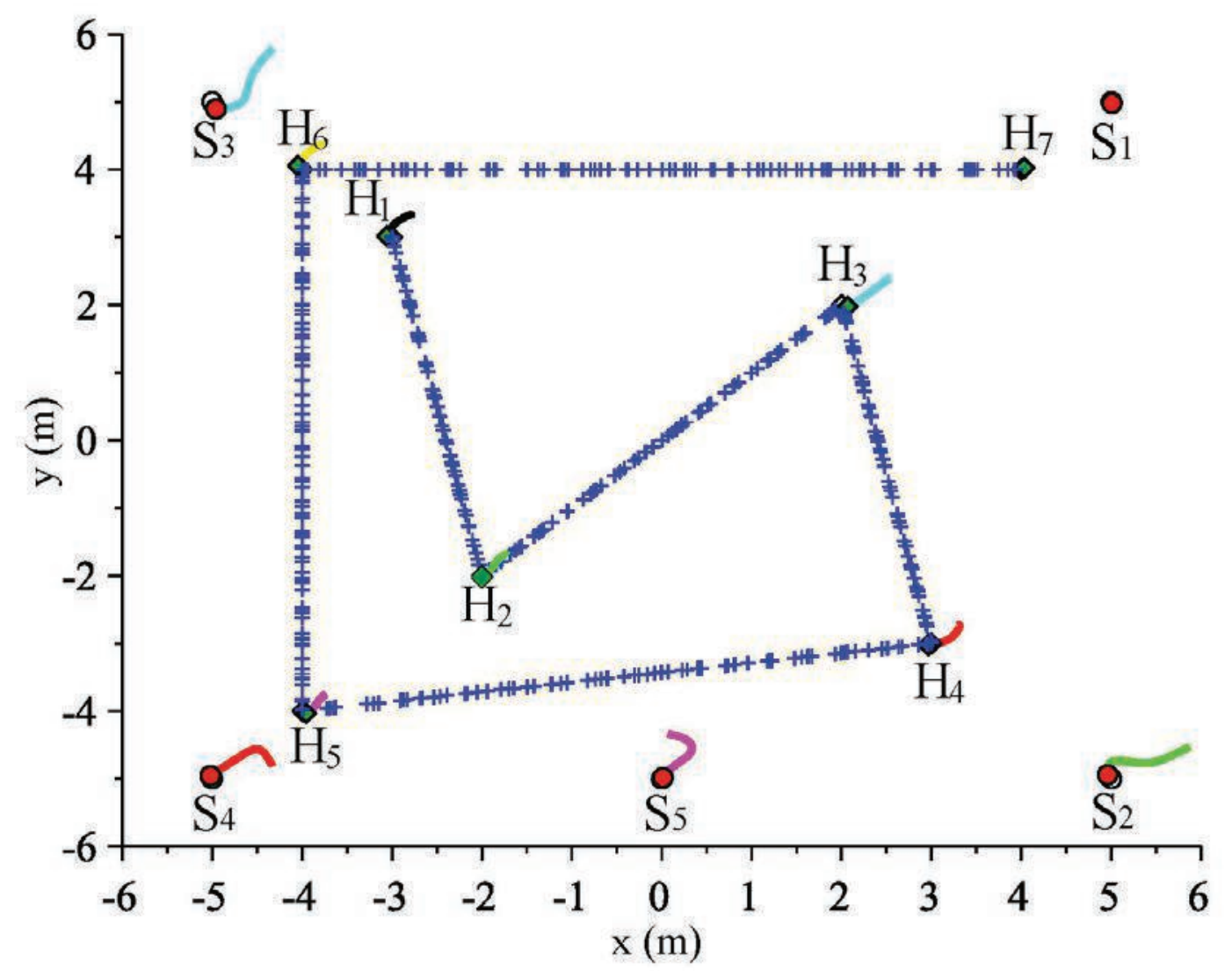

Fig. 5: Simulation results of the localization and self-calibration of the system. The standing positions and the positions of the sender are estimated from the data.

\section{Conclusion}

The synchronization enables high precision indoor localization. We show an accuracy of $0.4 \mathrm{~mm}$ for direct distance measurement for $10 \mathrm{~dB}$ SNR. Moreover, due to the self-calibration and the photovoltaic powered senders, the installation costs are low. Further, we showed the usage of OFDM to shorten the pulse length and therefore, reduce the interference by echoes. Consequently, we receive the line of sight signal without disturbance and achieve high precision localization.

The local optimization algorithms have been proved to be capable of locating with low error the sender positions, their offsets and the standing still receiver locations. The only requirement is to have a rough idea of the sender positions. The period of time when the receiver is not moving is successfully detected in the presence of Gaussian noise with the aid of an unscented Kalman filter. After this initialization step with the local optimization, the position can be tracked by recursive state estimation algorithms.

\section{Acknowledgement}

This work has been partly supported by the "Spitzencluster MicroTec Suedwest" and German Federal Ministry of Education and Research (BMBF) with the funding number $16 S V 5988$. 


\section{References}

[1] A. Ens, F. Höflinger, J. Wendeberg, R. Zhang, J. Hoppe, A. Bannoura, L. M. Reindl, and C. Schindelhauer, "Acoustic Self-calibrating System for Indoor Smartphone Tracking (ASSIST)," in International Journal of Navigation and Observation, vol. 2015, no. 694695, pp. 1-14, 2015.

[2] "ISO 9613-1, Acoustics - Attenuation of sound during propagation outdoors- Part1: Calculation of the absorption of sound by the atmosphere," ISO, 1993.

[3] R. Lerch, G. Sessler, D. Wolf. "Technische Akustik: Grundlagen und Anwendungen," Springer, Berlin, 2008.

[4] A. Glenn, "Comparison of PSK vs FSK and PSK-AM vs FSK-AM binary-coded transmission systems," IRE Transactions on Communications Systems, vol. 8, no. 2, pp. 87-100, Jun. 1960.

[5] M. Miller and J. Lee, "BER expressions for differentially detected pi;/4 DQPSK modulation," IEEE Transactions on Communications, vol. 46, no. 1, pp. 71-81, 1998.

[6] J. A. C. Bingham, "Multicarrier modulation for data transmission: an idea whose time has come," IEEE Communications Magazine, vol. 28, no. 5, pp. 5-14, 1990.

[7] G. Shen, R. Zetik, and R. S. Thoma, "Performance Comparison of TOA and TDOA Based Location Estimation Algorithms in LOS Environment," Proceedings of the 5th workshop on positioning, navigation and communication 2008 (WPNC 08), pp. 71-78, 2008.

[8] J. Wendeberg, J. Müller, C. Schindelhauer, and W. Burgard, "Robust Tracking of a Mobile Beacon using Time Differences of Arrival with Simultaneous Calibration of Receiver Positions," in Proceedings of 2012 International Conference on Indoor Positioning and Indoor Navigation (IPIN), 2012.

[9] F. Gustafsson and F. Gunnarsson, "Positioning using time-difference of arrival measurements," in Acoustics, Speech, and Signal Processing, 2003. Proceedings (ICASSP'03). 2003 IEEE International Conference, vol. 6, 2003, pp. VI-553.

[10] C. Hongyang, D. Ping, X. Yongjun, and L. Xiaowei, "A Robust Location Algorithm with Biased Extended Kalman Filtering of TDOA Data for Wireless Sensor Networks," in 2005 International Conference on Wireless Communications, Networking and Mobile Computing, 2005. Proceedings., vol. 2. IEEE, 2005, pp. 883-886.

[11] R. Zhang, F. Höflinger, and L. Reindl, "TDOA based localization using Interacting Multiple Model estimator and ultrasonic transmitter/receiver," in Systems, Signals and Devices (SSD), 2012 9th International MultiConference on, march 2012, pp. 1-6.

[12] J. Wendeberg, F. Höflinger, C. Schindelhauer, und L. Reindl, "Calibration-Free TDOA Self-Localization," Journal of Location Based Services, 2013.

[13] J. Bordoy, P. Hornecker, J. Wendeberg, R. Zhang, C. Schindelhauer, und L. Reindl, "Robust tracking of a mobile receiver using unsynchronized time differences of arrival," in IEEE 2013 International Conference on Indoor Positioning and Indoor Navigation (IPIN), 2013.

[14] M. Freunek, M. Freunek, and L. Reindl, "Maximum efficiencies of indoor photovoltaic devices," IEEE Journal of Photovoltaics, vol. 3, pp. 59-64, Jan. 2013.

[15] A. Ens und L. M. Reindl, "Piezoelectric transceiver matching for multiple frequencies," Journal of Sensors and Sensor Systems, vol. 4, no. 1, pp. 9-16, Jan. 2015.

[16] A. Ens, T. Janson, L. M. Reindl and C. Schindelhauer, "Robust multicarrier frame synchronization for localization systems with ultrasound," in Proc. of the 18th International OFDM Workschop (InOWo'14) 2014, Essen, Germany, Aug. 2014. 